\title{
Efficacy and Safety of Reinforced Versus Standard Vaccine Schedule Towards Hepatitis B In Chronic Kidney Disease: A Systematic Review and Meta-Analysis
}

\author{
Fabrizio Fabrizi, ${ }^{1,}{ }^{*}$ Francesca Maria Donato, ${ }^{2}$ and Piergiorgio Messa $^{3}$ \\ ${ }^{1}$ Division of Nephrology, Maggiore Hospital and IRCCS Foundation, Milano \\ ${ }^{2}$ Division of Gastroenterology, Maggiore Hospital and IRCCS Foundation, Milano \\ ${ }^{3}$ Division of Nephrology, Maggiore Hospital, University School of Medicine, Milano \\ "Corresponding author: Fabrizio Fabrizi, Division of Nephrology, Maggiore Hospital, IRCCS Foundation, Pad. Croff, via Commenda 15, 20122 Milano, Italy. Tel: +39-255034552, \\ Fax:+39-255034550, E-mail: fabrizi@policlinico.mi.it
}

Received 2016 November 21; Accepted 2016 December 11.

\begin{abstract}
Context: The immunogenicity of the hepatitis B virus vaccine is reduced in patients with renal failure compared with the nonuraemic population. A variety of approaches have been suggested to improve the immune response in uraemic population including an increase in dose of the hepatitis B vaccine.

Objective: To compare the efficacy and safety of hepatitis B vaccine schedules based on greater versus standard doses of $\mathrm{HB}$ vaccine in patients with chronic kidney disease stages 3 - 5 .

Evidence Acquisition: We carried out a systematic review of the medical literature with a meta-analysis of randomized trials comparing seroprotection rates after greater vs. standard doses of the HB vaccine. The odds ratio to obtain seroprotection among patients who received greater (study group) vs. standard (control group) doses was the end-point of interest. We used a random-effects approach, as described by DerSimonian and Laird, with heterogeneity and subgroup analyses.

Results: We retrieved 11 clinical trials ( $n=870$ unique patients); $2(n=141$ patients) and 8 studies $(n=689)$ included CKD patients on pre-dialysis and dialysis stage, respectively. Three trials $(n=368$ patients) employed plasma-derived vaccine; $8(n=502)$ adopted recombinant vaccine. Aggregation of study results ( $\mathrm{n}=10$ studies) showed that the seroprotection rate (short-term follow-up) towards $\mathrm{HB}$ virus was higher among patients receiving greater than standard doses of vaccine [pooled OR, 2.10, 95\% confidence intervals, 1.15 -3.82]. The P-value was 0.0001 for our test to study heterogeneity. The seroprotection rate towards HBV was much greater in the subset of trials $(\mathrm{n}=2)$ based on plasma-derived vaccine (OR, 3.78; 95\% CI, 2.35; 6.07), and no heterogeneity was found (NS). In the subset of RCTs ( $\mathrm{n}=8$ studies), the seroprotection rate was higher among patients receiving greater doses of vaccine towards HBV, OR, 2.01 (95\% CI, 0.92; 4.39), with significant heterogeneity $(\mathrm{P}=0.002)$. Tolerance was satisfactory and no dropouts due to side effects were reported.

Conclusions: Vaccine schedules based on greater than standard doses of HB vaccine offer higher immunogenicity in patients with chronic kidney disease. These results support the current recommendations to give higher doses of HBV vaccine to susceptible dialysis population in order to increase the sero-protection rate. Further research is needed to assess whether these findings apply to $\mathrm{HB}$ vaccines provided with novel adjuvants.
\end{abstract}

Keywords: Chronic Kidney Disease, Dialysis, Hepatitis B, Meta-Analysis, Seroprotection, Vaccine

\section{Context}

On a global basis, hepatitis B is one of the most important infectious diseases all over the world. The control of the spread of the hepatitis B virus infection in dialysis population has been an important goal in the management of end-stage renal disease (1). Numerous multicenter surveys have shown a low but not negligible frequency of HBV infection in dialysis units of developed countries (2-4). On the contrary, prevalence and incidence rates of HBV remain high within dialysis facilities of the developing world (5). In addition, outbreaks of the HBV infection among patients undergoing long-term hemodialysis con- tinue to be reported in industrialized countries (6). To prevent transmission of $\mathrm{HBV}$ in haemodialysis units, numerous measures have been performed including the screening of blood for the hepatitis B surface antigen (HBsAg), the decline in the number of blood transfusions, and the implementation of universal and specific measures within dialysis rooms, as recommended by the centers for disease control and prevention (CDC, Atlanta, Georgia, USA) (7). The hepatitis $B$ vaccination is another factor responsible for the decline in HBsAg incidence rates among dialysis patients over recent years.

Patients with chronic kidney disease and renal fail- 
ure typically show an impaired immune response to the hepatitis B virus vaccine (1). They have lower seroprotection rates compared with healthy subjects; moreover, after completion of vaccination schedule anti-HBs titers of responder uraemic patients are low and decline logarithmically over time (1). Various approaches have been made to overcome the non-responsiveness of chronic uraemic patients including co-administration of adjuvants (8), or immune modulators (9), intradermal administration of $\mathrm{HB}$ vaccine (10), increased vaccine doses, or additional inoculations (11). Alternatively, it has been recommended to start vaccination in the early stages of a renal disease (12), when one could anticipate that the primary immune response is still satisfactory.

\section{Objective}

The goal of this study was to investigate the available evidence on the efficacy and safety of increased versus standard hepatitis $B$ vaccine doses in patients with renal failure by performing a systematic review of the literature. A meta-analysis of randomized and quasi-randomized trials comparing greater vs. standard hepatitis B vaccine doses in patients with chronic kidney disease stages 3 - 5 has been made.

\section{Methods}

This work is in agreement with the Preferred Reporting Items for Systematic reviews and Meta-Analyses statement (PRISMA guidelines) (see supplementary File 1) (13).

\subsection{Search Strategy and Data Extraction}

Electronic searches of the National Library of Medicine's MEDLINE database, current contents, and manual searches of selected speciality journals were performed to identify all pertinent literature. It has previously demonstrated that an electronic search alone may not be sensitive enough (14). We conducted our search by 4 MEDLINE dabase engines (Embase, Grateful Med, Ovid, and Pubmed); the cochrane library was also used.

We applied the following algorithm in medical subject heading and in free text words: "HEPATITIS B" or "HEPATITIS B VIRUS INFECTION" or "HBsAg POSITIVE STATUS"), ("CHRONIC KIDNEY DISEASE" or "CKD” or "END-STAGE RENAL DISEASE" or "ESRD" or "RENAL FAILURE" or "RENAL INSUFFICIENCY" or "RENAL IMPAIRMENT"), ("VACCINE" or "VACCINATION" or "ACTIVE IMMUNIZATION"), and ("ODDS RATIO" or "OR”). Reference lists from qualitative topic reviews and published clinical trials were also searched. Our search was limited to human studies that involved individuals aged $>18$ years published in the English literature. All articles were identified by a search from 1982 through September 1, 2016.

\subsection{Data Extraction and Assessment of Risk of Bias}

Data extraction was conducted independently by 2 investigators (F.F., F.M. D.) by using standardized data extraction forms. Studies were compared to eliminate duplicate reports for the same patients, which included contact with investigators when necessary. Wherever duplicate studies were found, the most complete report was included. Eligibility and exclusion criteria were prespecified. Disagreements were managed by consensus.

The risk of bias was independently assessed by 2 authors (F.F., F.M. D.) according to the recommendations regarding the risk of bias assessment tools for use in RCTs and nonrandomized studies (15).

\subsection{Criteria for Inclusion}

To be included in this systematic review, a clinical trial had to fulfil a set of criterias. It had to be published as a peer-reviewed paper; we included only prospective, randomized or quasi-randomized clinical trials comparing the sero-protection rate after schedules based on greater versus standard hepatitis B virus vaccine dose in adults with chronic kidney disease (stages 3 -5). The patients were followed for a minimum of 7 months after the first vaccine shot. The decision as to inclusion or exclusion of clinical trials was not related to the results. Patients who underwent a primary vaccination schedule (naïve patents), or those who failed to respond to a prior vaccine schedule (non-responder patients) against hepatitis B were enrolled. Hepatitis $B$ vaccines of all types and doses have been considered. Studies restricted to students, military recruits, or other cohorts that involved subjects $<19$ years of age were excluded.

\subsection{End-Points of Interest}

We compared the seroprotection rate at the completion of the vaccination course and over follow-up in adult patients with CKD stages 3-5 who received greater or standard doses of $\mathrm{HB}$ vaccine by intramuscular route. Seroprotection rate over follow-up was the frequency of responder patients who showed seroprotective antibody 12 months after completing the vaccine schedule. The seroprotection rate to develop protective antibody titers towards HBsAg (anti-HBs) after HB vaccination was the primary end-point (at completion of vaccine schedule and over follow-up). Secondary end-point included adverse events of the hepatitis B vaccine (local injection site and systemic reactions, liver-related morbidity and mortality). 


\subsection{Ineligible Studies}

Studies were excluded if they reported inadequate data on measures of response. Reports that were published in abstract form, or as interim reports were excluded; review articles were not considered for this analysis. Studies including patients receiving immunosuppressive drugs, or with malignant disease and human immunodeficiency virus infection were not enrolled. Clinical trials concerning renal transplant patients were excluded.

\subsection{Definitions}

In all trials included in this analysis, data from patients who did not complete the vaccination program were excluded from the final analysis; thus, analysis was made perprotocol, not by ITT. When not provided in the publication, the outcome, according to the per-protocol method, was calculated by the data abstractors (F.F., and F.M.D.). In all studies, the vaccine towards HB was administered as an intramuscular injection into the deltoid region of the arm without or least likely to be used for the arteriovenous fistula in hemodialysis and pre-dialysis patients or the nondominant arm in peritoneal dialysis patients.

Primary outcome measure in this systematic review was the seroprotection and seroconversion rate. Seroprotection was defined as the frequency of patients developing protective titres (patients with anti-HBs titres $>10$ $\mathrm{mIU} / \mathrm{mL}$ ) when previously negative. Seroconversion was defined as the development of detectable antibody towards the hepatitis B surface antigen (patients with antiHBs titres $>1 \mathrm{mIU} / \mathrm{L}$ ) when previously negative. Seroprotection and seroconversion rates were calculated at completion of vaccination schedule and over follow-ups. These definitions were consistent with standards published in the scientific literature.

\subsection{Statistical Methods}

The ORs were generated using the random effects model. The random effects approach was made according to DerSimonian and Laird (16). The Cochrane's Q-test was used for quantifying the heterogeneity; a value $<0.10$ was considered indicative of a statistically significant difference (17). In addition, the consistency of effects across studies was evaluated by I2 index (18). A sensitivity analysis using a fixed-effects model was also performed to assess the consistency of results. The Galbraith plot was used to assess the heterogeneity and precision of single studies (19). Predefined subgroup analyses were conducted to calculate pooled ORs (sensitivity analyses). The publication bias assessment (number of void or negative trials necessary to render the meta-analysis meaningless) was calculated according to the Klein formula (20). The publication bias was also measured by funnel plot asymmetry test. Every estimate was given with 95\% Confidence Intervals. The $5 \%$ significance level was used for $\alpha$-risk. All the statistical analyses were performed using the Stata 8.0 software (Stata Corporation, College Station, TX, US).

\section{Results}

\subsection{Literature Review}

Our electronic and manual searches identified 797 studies, of which 431 were considered potentially relevant and were selected for full text review (Figure 1). A total of 11 studies met the inclusion criteria (21-31). A complete list of the 431 reports reviewed is available (see supplementary File 2). There was a $100 \%$ concordance between reviewers with respect to final inclusion and exclusion of studies reviewed based on the predefined inclusion and exclusion criteria.

\subsection{Study Quality and Risk of Bias}

The majority of studies were RCTs; a few $(\mathrm{n}=3)$ studies had a comparative design. All studies were published in the English language from 1990 to 2010. The risk of bias was unclear in all included studies, as the study methodology was incompletely reported; generation of allocation sequence was given in 3 studies $(21,29,31)$ and blinding of participants and personnel was mentioned in 1 (22).

No studies were assessed as having great methodological quality (or reduced risk of bias). The risk of reporting bias was low in all studies as the measures of the primary outcome were objective. The risk of bias regarding 1 ) random sequence generation, 2) allocation concealment, 3) blinding, and 4) incomplete outcome data, remained unclear across most studies (see supplementary File 3). Stratified analysis showed no differences in primary outcomes between studies provided with better quality (RCTs) and those with comparative design (CCTs) (see below).

\subsection{Patient Characteristics}

Some salient demographic and clinical characteristics of subjects enrolled in the RCTs of the current metaanalysis are shown in Tables 1-3. There were 6 reports from Europe. The mean age of subject cohorts ranged from 39.9 \pm 14 to 66 (30-81) years, and the rate of males was $66 \%$. A minority of patients $(n=141)$ had chronic kidney disease at the pre-dialysis stage $(24,29)$; patients on peritoneal dialysis ( $\mathrm{n}$ $=139$ ) were included in only 2 studies $(30,31)$. Three studies employed plasma-derived vaccines; 8 studies recombinant vaccines, Engerix-B, Glaxo-Smith Kline $(n=6)$, and Recombivax, MSD $(n=1)$. Data on vaccine schedule in the RTCs included in this meta-analysis are shown in Table 3. 


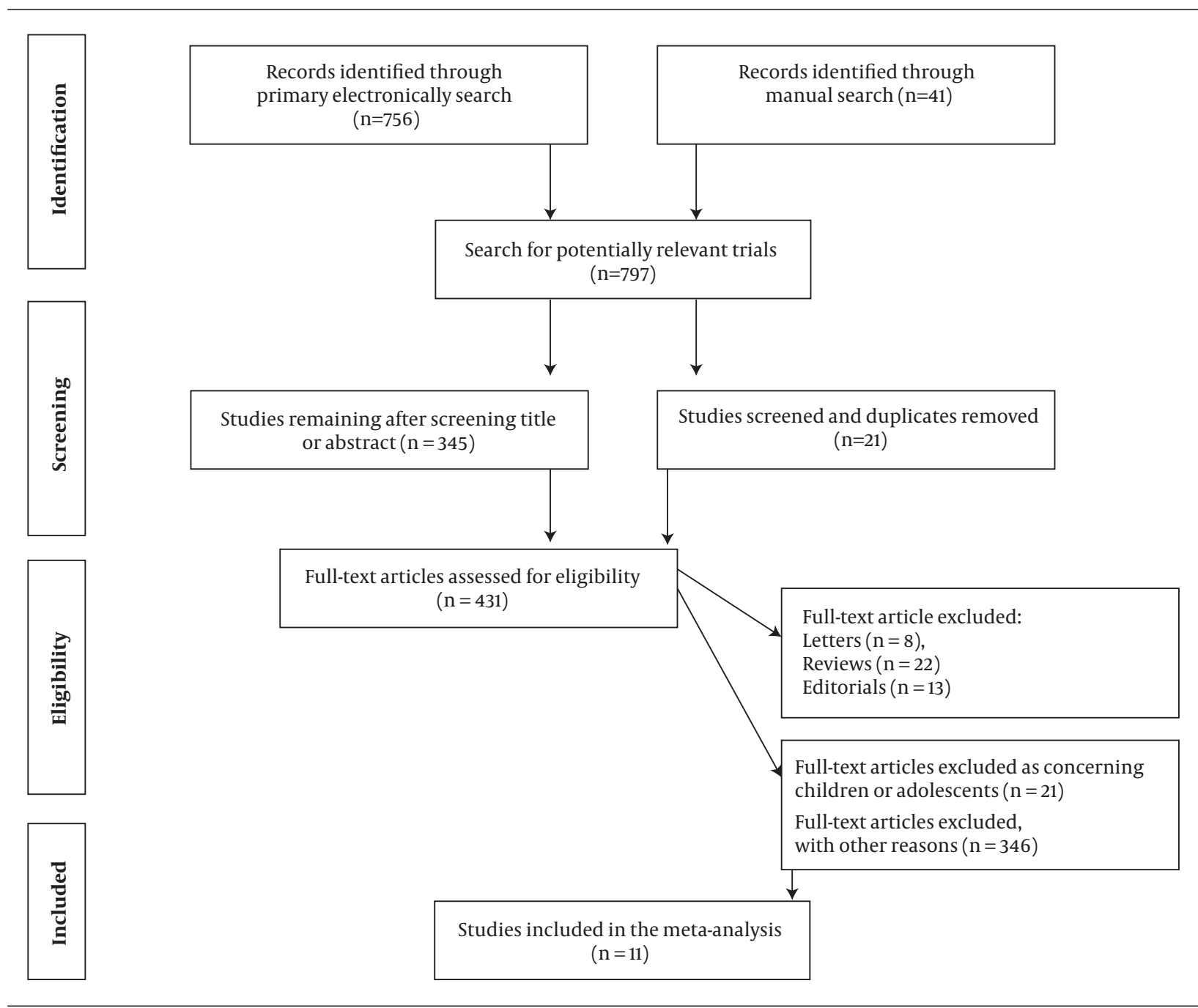

Figure 1. Flow Diagram of the Literature Search-Process

\subsection{Summary Estimates of Outcome}

Aggregation of study $(n=10)$ results showed that the seroprotection rate (short-term follow-up) towards HB virus was greater among patients receiving greater vaccine doses [pooled OR, 2.10, 95\% confidence intervals (95\% CI), 1.15 - 3.82]. The publication bias assessment, according to the Klein formula, was 51 . The test of funnel plot asymmetry was not significant, $\alpha=0.01 ; 95 \% \mathrm{CI},-3.96 ; 3.97, \mathrm{P}(\mathrm{z})=$ 1.00 (Figure 2). Figure 3 shows the seroprotection rate according to the Peto method; the test for heterogeneity was significant. The Galbraith plot (Figure 4) highlighted the great precision of every single study, and the occurrence of heterogeneity in the analysis.

Subgroup analyses were undertaken to explain the heterogeneity across trials (Table 4). In the subset of patients on dialysis, the risk to develop seroprotection towards $\mathrm{HB}$ virus was greater among patients on standard doses of vaccine, pooled OR, $1.96(0.86 ; 4.47)$ (Table 4$)$. The test for heterogeneity was significant (Table 4). Only 4 studies (23, 24, $30,31)$ gave information on the seroprotection over a longterm follow-up (Table 4). The risk of seroprotection among patients receiving reinforced vs. standard vaccine doses in European trials and over long-term follow-up is shown in Figures 5 and 6.

\subsection{Side-Effects}

Information on adverse events was given in 6 studies (23-25, 29-31). Two hepatitis B infections occurred over the follow-up in 1 trial (21), no SAEs were observed in the others (23-25, 29-31). No dropouts due to side effects were reported. Many RCTs reported minor side effects including local (soreness at injection site) and general (low-grade 
Table 1. Baseline Characteristics of Studies Included in the Analysis ${ }^{\mathrm{a}}$

\begin{tabular}{|c|c|c|c|c|}
\hline Authors & Country & Patients, $\mathbf{n}$ & Publication Year & CKD Stage \\
\hline Benhamou E, et al. & France & $65 / 68$ & 1984 & Dialysis \\
\hline Aronoff G, et al. & US & $13 / 11$ & 1985 & Dialysis \\
\hline Lelie N, et al. & The Netherlands & $106 / 105$ & 1985 & Dialysis \\
\hline Seaworth B, et al. & US & $16 / 19$ & 1988 & Pre-dialysis \\
\hline Bruguera $M$, et al. & Spain & $37 / 65$ & 1989 & Dialysis \\
\hline Mitwalli A, et al. & Saudi Arabia & $17 / 15$ & 1996 & Dialysis \\
\hline Mauri M, et al. & Spain & $30 / 10$ & 1997 & NA \\
\hline Ayli D, et al. & Turkey & $30 / 30$ & 2000 & Dialysis \\
\hline McNulty C, et al. & UK & $55 / 51$ & 2005 & Pre-dialysis \\
\hline Chow K, et al. & Hong Kong & $26 / 14$ & 2006 & Dialysis \\
\hline Chow K, et al. (2) & Hong Kong & $45 / 42$ & 2010 & Dialysis \\
\hline
\end{tabular}

${ }^{\mathrm{a}}$ Data are given for study/control patients where appropriate.

Table 2. Baseline Characteristics of Studies Included in the Analysis ${ }^{\mathrm{a}}$

\begin{tabular}{|c|c|c|c|c|}
\hline Authors & Age (Years) & Male, $\mathbf{n}$ & Time on Dialysis (mo) & Dialysis Mode \\
\hline Benhamou E, et al. & $48.8 \pm 1 / 52 \pm 1$ & $46(67 \%) / 35(50 \%)$ & $27.6 \pm 2 / 22.8 \pm 1$ & HD \\
\hline Aronoff G, et al. & $54.5 \pm 8 / 52.1 \pm 7$ & $24(100 \%)$ & NA & HD \\
\hline Lelie N, et al. & $54.4 \pm 1 / 56 \pm 1$ & $59(51 \%) / 69(61 \%)$ & NA & HD \\
\hline Seaworth B, et al. & $46.1 / 44.6$ & $15(75 \%) / 14(67 \%)$ & NA & Pre-dialysis \\
\hline Mitwalli A, et al. & $39.9 \pm 14$ & $18(57 \%)$ & NA & $\mathrm{HD}(\mathrm{n}=27,64 \%)$ \\
\hline Mauri M, et al. & NA & NA & NA & NA \\
\hline Ayli D, et al. & $41.4 \pm 2.4$ & $34(57 \%)$ & $11.8 \pm 1.1$ & HD \\
\hline McNulty C, et al. & $64(31-85) / 66(30-81)$ & $40(62.5 \%) / 35(61 \%)$ & NA & Pre-dialysis \\
\hline Chow K, et al. & $43 \pm 12$ & $21(51 \%)$ & $8.5(1-33)$ & $\operatorname{PD}(n=52,81 \%), \mathrm{HD}(\mathrm{n}=12,19 \%)$ \\
\hline
\end{tabular}

Abbreviation: NA, not available.

${ }^{a}$ Data are given for study/control patients where appropriate.

fever, fatigue, and mild headache) events. The incidence of minor side effects ranged between 15\% (24) and 10.3\% (25). Reporting was not performed by treatment arm and we could not assess differences between study vs. control groups. One RCT reported that local reactogenicity was greater with reinforced than standard vaccine schedules (23) (see supplementary File 4).

\section{Discussion}

Many clinical and background factors have been linked with the poor immunogenicity of $\mathrm{HB}$ vaccines in patients with chronic kidney disease compared with healthy individuals such as age (32), male gender (33), nutritional status (34), serological positivity for hepatitis C virus (35) or human immunodeficiency virus (36) infection, diabetes mellitus (37), blood transfusion history (38), and possession of the major histocompatibility complex aplotype HLA-B (39) among others. Finally, the failure to complete a full course of $\mathrm{HBV}$ vaccination may cause a poor active immunization (40). In addition, numerous in vivo and in vitro experiments have shown that the impaired response to the HBV vaccine in uremic individuals has been related to additional factors: a lower activation of T-lymphocytes (naïve CD4) to helper cells by overproduction of TNF- $\alpha$, re- 
Table 3. Details of Vaccination Schedule in the Clinical Trials Included in the Analysis ${ }^{\mathrm{a}}$

\begin{tabular}{|c|c|c|c|c|}
\hline Authors & Vaccine & Vaccine Schedule & Study Design & Vaccine Dose \\
\hline Benhamou E, et al. & Plasma & $0,1,2$ by SC & RCT & $2 \mathrm{~mL} \times 3 / 1 \mathrm{~mL} \times 3$ \\
\hline Aronoff G, et al. & Plasma & $0,1,6$ by IM & RCT & $40 \mathrm{mcg} \times 3 / 20 \mathrm{mcg} \times 3$ \\
\hline Lelie N, et al. & Plasma & $0,1,2$ by IM & RCT & $27 \mathrm{mcg} \times 3 / 3 \mathrm{mcg} \times 3$ \\
\hline Seaworth B, et al. & Recombinant & $0,1,6$ by IM & RCT & $40 \mathrm{mcg} \times 3 / 20 \mathrm{mcg} \times 3$ \\
\hline Bruguera $M$, et al. & Recombinant & $0,1,2,6$ by IM & Prospective, CCT & $40 \mathrm{mcg} \times 4 / 20 \mathrm{mcg} \times 4$ \\
\hline Mitwalli A, et al. & Recombinant & $0,1,2$ by IM & Prospective, CCT & $2 \mathrm{~mL} \times 3 / 1 \mathrm{~mL} \times 3$ \\
\hline Mauri M, et al. & Recombinant & $0,1,2,6$ by IM & RCT & $40 \mathrm{mcg} \times 4 / 20 \mathrm{mcg} \times 4$ \\
\hline Ayli D, et al. & Recombinant & $0,1,6,9$ by IM & RCT & $40 \mathrm{mcg} \times 4 / 20 \mathrm{mcg} \times 4$ \\
\hline McNulty C, et al. & Recombinant & $0,1,6$ by IM & RCT & $40 \mathrm{mcg} \times 3 / 20 \mathrm{mcg} \times 3$ \\
\hline Chow K, et al. & Recombinant & $0,1,6$ by IM & Retrospective, cohort & $40 \mathrm{mcg} \times 3 / 20 \mathrm{mcg} \times 3$ \\
\hline Chow K, et al. (2) & Recombinant & $0,1,6$ by IM & RCT & $80 \mathrm{mcg} \times 3 / 40 \mathrm{mcg} \times 3$ \\
\hline
\end{tabular}

${ }^{\mathrm{a}}$ Data are given for study/control patients where appropriate.

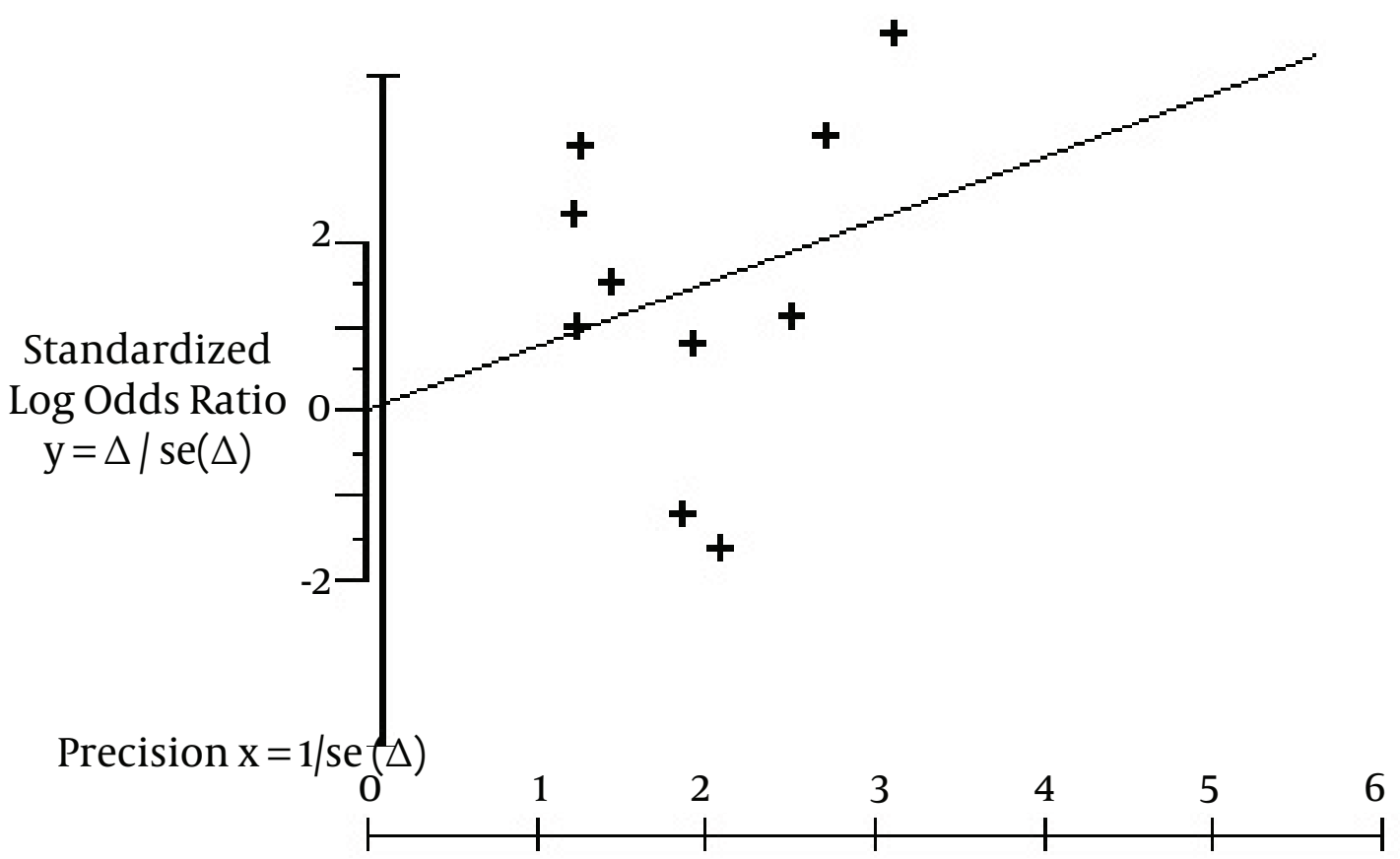

Figure 2. Risk to Obtain Seroprotection: Reinforced Versus Standard Dose of Hepatitis B Vaccine (Test of Funnel Plot Asymmetry) (All Trials)

duced synthesis of interleukin-2 (and therefore enhanced expression of IL-2 receptors), and reduced TCR/CD3 antigen receptor complex. Low T cell activation prevents B cells from producing adequate amounts of antibody to the T- processed antigen.

According to our systematic review, reinforced schedules of the $\mathrm{HB}$ vaccine enhance the response rate in uraemic patients. This phenomenon is particularly clear 


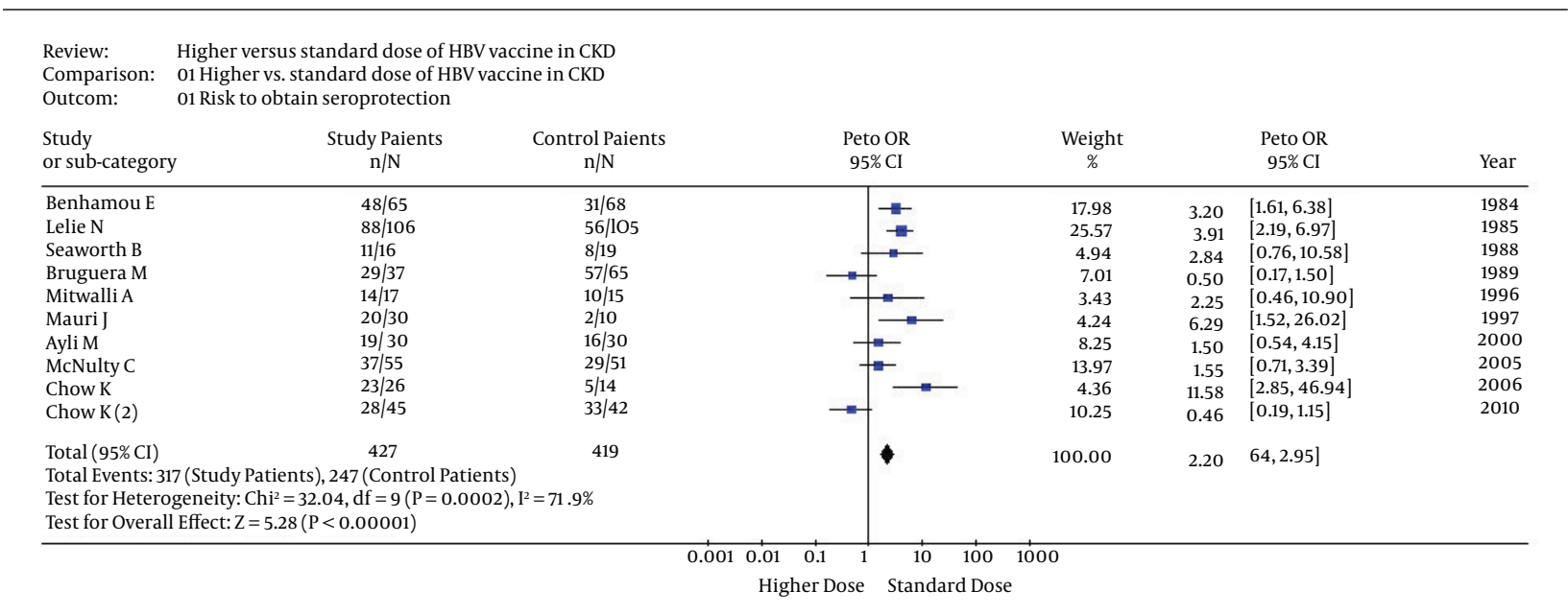

Figure 3. Risk to Obtain Seroprotection: Reinforced Versus Standard Dose of Hepatitis B Vaccine (Forest Plot) (All Trials)

Table 4. Pooled Odds Ratio (OR) to Respond to HB Vaccination (Standard Versus Greater Vaccine Doses) in Various Subgroups of Interest

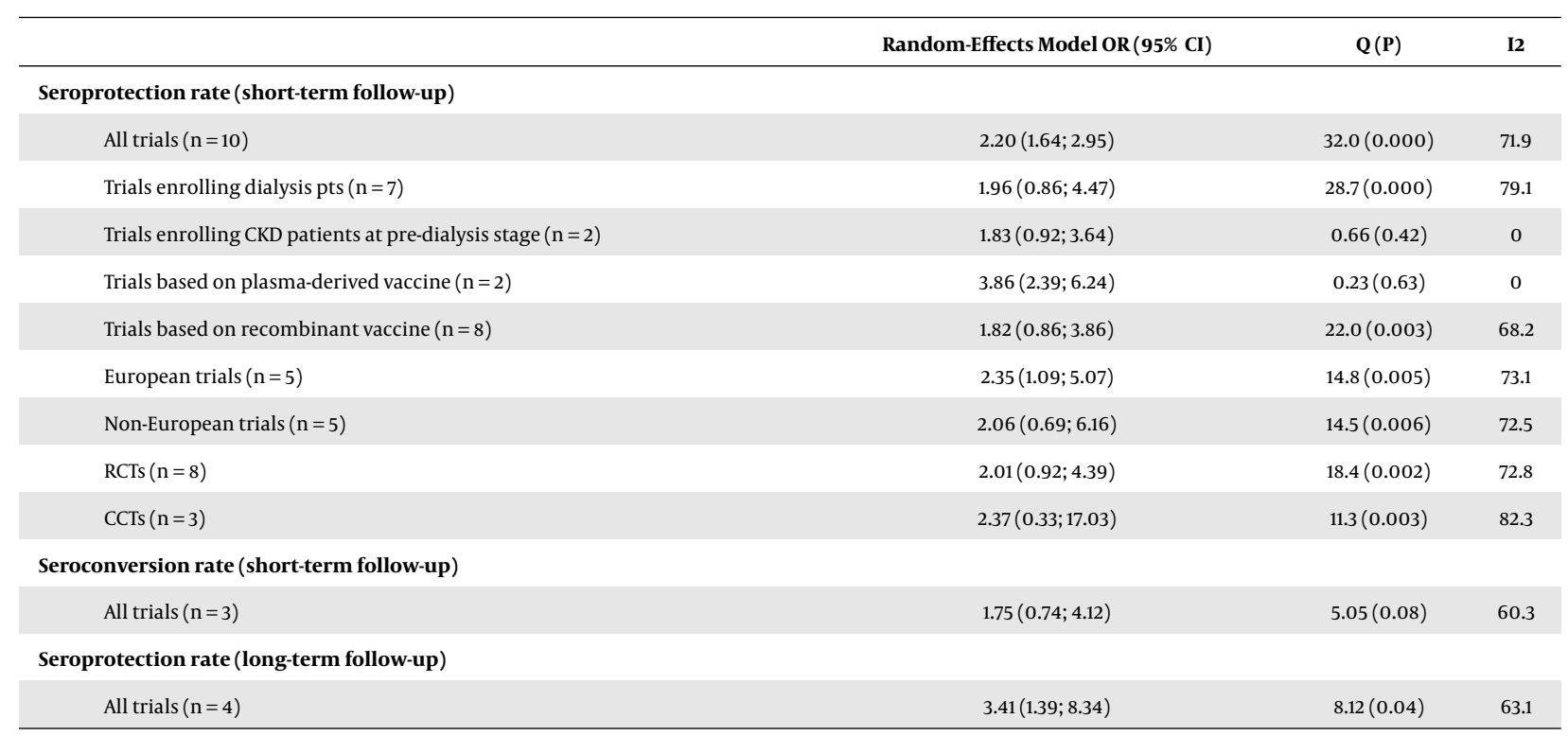

in the subset of trials using plasma-derived vaccines; an increased immunogenicity of $\mathrm{HB}$ vaccine with reinforced schedule appears in the subset of trials based on recombinant vaccine, even if no statistical association was found. These results are in keeping with the guidelines from the CDC, which recommend greater doses of the recombinant hepatitis B virus vaccine (Engerix-B) and increased number of vaccine shots for active immunization of patients on maintenance dialysis (40 mcg; 0,1,2, and 6 months)(7). The vaccine schedule recommended for pre-dialysis patients consists of 3 shots with standard doses of hepatitis B virus vaccine (20 mcg; 0,1, and 6 months)(7). A recent systematic review concluded that double-dose vaccination for CKD patients did not improve seroconversion compared to patients on standard HBV vaccine; the lower number of studies could be implicated in these conflicting findings (41).

Vaccination towards HBV has played a crucial role in the decline in HBsAg incidence rates among dialysis patients in the developed world over recent years (7). Recent evidence has been accumulated regarding the additional benefits given by vaccination towards $\mathrm{HBV}$; these include the possibility that HBsAg negative patients with sero-protective levels of antibody against HBV (HBsAb >10 $\mathrm{mIU} / \mathrm{mL}$ ) can undergo haemodialysis in shifts and rooms 


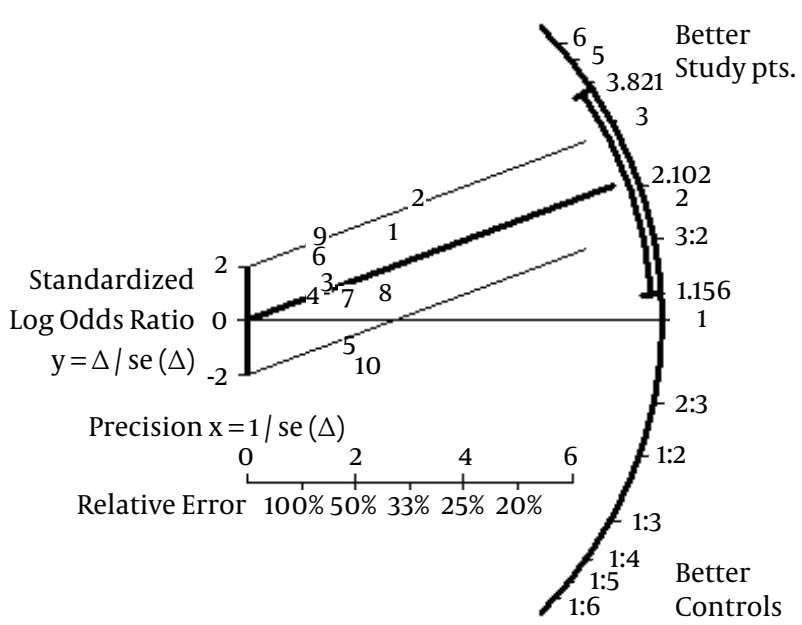

Figure 4. Risk to Obtain Seroprotection: Reinforced Versus Standard Dose of Hepatitis B Vaccine (Galbraith Plot) (All Trials)

dedicated for HBsAg positive patients. Isolation of HBsAg positive carriers by rooms, machines, and staff remains an important preventive measure against the spread of HBV within dialysis units, as recommended by the CDC(7). Seroprotection against HBV is crucial to RT candidates in order to be included in the waiting list from anti-HBc positive kidney donors. Finally, it appears that vaccination towards HBV in patients with CKD is an effective measure to reduce the progression of chronic kidney disease. A significant link between HBsAg positive serologic status and increased risk for end-stage renal disease has been recently noted (42).

As with all systematic reviews, this study has several limitations, an examination of which may inform the design and conduct of future studies on this topic. First, despite stringent inclusion criteria, the methodological quality of the studies was on average not ideal. Among the 11 studies included in this analysis, some did not describe methods of randomization and double-blinding was uncommon; it is possible that some so called 'randomized controlled trials' were not real randomized controlled trials owing to a lack of rigorous clinical trial design (43). In the context of a systematic review, the ratings of the quality of evidence reflect the extent of our confidence that the effect estimates are correct (44). However, pooling the subgroup of trials provided with more rigorous clinical trial designs did not significantly change our findings. Secondly, individual data (e.g., "patient-level" data) from each study were not available; thus, it was impossible to perform our own adjustments. Thirdly, as with all metaanalyses, this study has the potential limitation of publication bias; negative trials are less likely to be published. To limit the possible effects of publication bias, we adopted various strategies to identify published and unpublished trials. Inclusion criteria, established a priori, were chosen to increase the likelihood that high-quality studies would be included. Finally, the number $(n=11)$ of studies included in this meta-analysis clearly precludes more definitive conclusions.

An additional limitation of this study concerns the use of anti-HBs titer as a surrogate marker of the protection against HBV infection. The protective effect of the recombinant vaccine against acquisition of HBV infection among patients on long-term dialysis was already reported by the CDC (45). The efficacy of reinforced versus standard doses of vaccine towards HBV should be evaluated in terms of reduced incidence of $\mathrm{HBV}$ infection in $\mathrm{CKD}$ populations. The very low incidence of $\mathrm{HBV}$ infection among patients on maintenance dialysis in the developed world clearly makes the implementation of such clinical trials difficult.

In summary, this meta-analysis of RCTs has shown a consistent benefit of reinforced versus standard schedules of the HB vaccine in patients with renal failure. Recent evidence has been gathered on adjuvanted recombinant $\mathrm{HB}$ vaccines (HBVAS04) $(46,47)$ or third-generation vaccines (48) (recombinant HBV vaccines expressed in mammalian cells containing S/Pre-S antigens) in chronic kidney disease population. Trials comparing efficacy and safety of adjuvanted HBVASO 4 vaccine versus recombinant vaccine (with greater doses) in patients with chronic kidney disease are under way.

\section{Supplementary Material}

Supplementary material(s) is available here.

\section{Acknowledgments}

The authors' work is supported in part by a 'Project Glomerulonephritis' grant, in memory of Pippo Neglia, by Associazione Amici del Croff-Onlus. The funders had no role in the study design, data collection analysis, decision to publish, or preparation of the manuscript.

\section{Footnote}

Financial Disclosure: None 


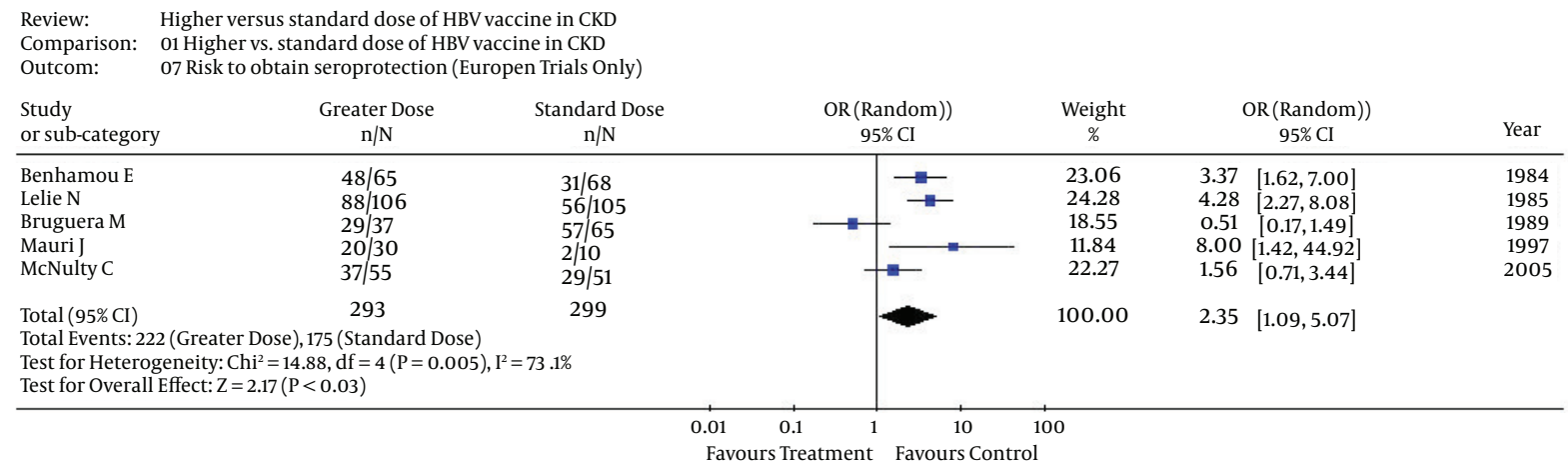

Figure 5. Risk to Obtain Seroprotection: Reinforced Versus Standard Dose of Hepatitis B Vaccine (Forest Plot) (European Trials Only)

Review: $\quad$ Higher versus standard dose of HBV vaccine in CKD

Comparison: 01 Higher vs. standard dose of HBV vaccine in CKD

Outcom: 10 Risk to obtain seroprotection (Long Term Follow-Up)

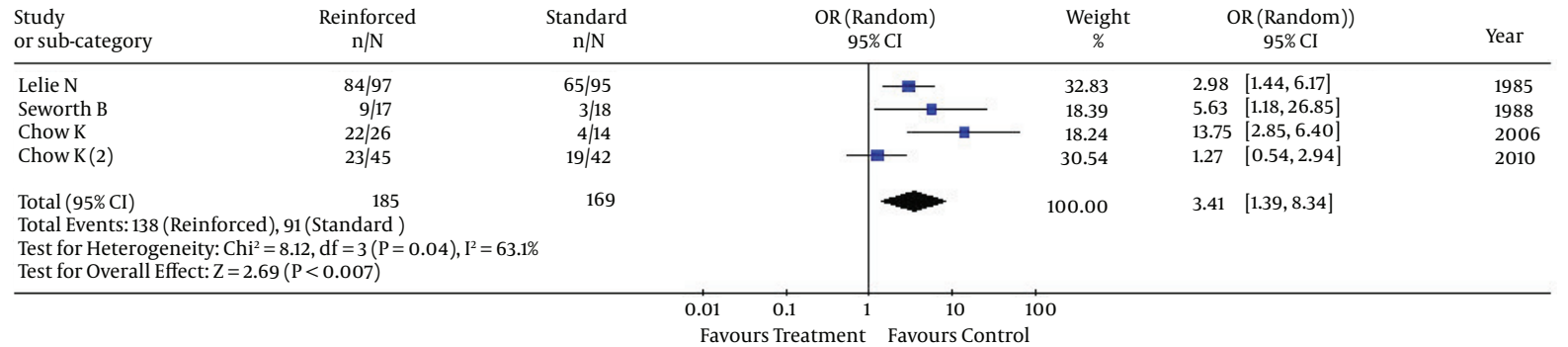

Figure 6. Risk to Obtain Seroconversion: Reinforced Versus Standard Dose of Hepatitis B Vaccine (Forest Plot) (Long-Term Follow-Up)

\section{References}

1. Fabrizi F, Martin P. Hepatitis B virus infection in dialysis patients. Am J Nephrol. 2000;20(1):1-11. [PubMed: 10644861].

2. Burdick RA, Bragg-Gresham JL, Woods JD, Hedderwick SA, Kurokawa $\mathrm{K}$, Combe $\mathrm{C}$, et al. Patterns of hepatitis B prevalence and seroconversion in hemodialysis units from three continents: the DOPPS. Kidney Int. 2003;63(6):2222-9. doi: 10.1046/j.1523-1755.2003.00017.x. [PubMed: 12753311].

3. Finelli L, Miller JT, Tokars JI, Alter MJ, Arduino MJ. National surveillance of dialysis-associated diseases in the United States, 2002. Semin Dial. 2005;18(1):52-61. doi: 10.1111/j.1525-139X.2005.18108.x. [PubMed: 15663766].

4. Garcia Agudo R, Aoufi Rabih S, Barril Cuadrado G, Proy Vega B, Arias Arias A, Herruzo Gallego JA, et al. Spanish multicentre PIBHE study: Prevalence and immunization of chronic hepatitis B in haemodialysis patients in Spain. Nefrologia. 2016;36(2):126-32. doi: 10.1016/j.nefro.2015.10.013. [PubMed: 26875043].

5. Johnson DW, Dent H, Yao Q, Tranaeus A, Huang CC, Han DS, et al. Frequencies of hepatitis $\mathrm{B}$ and $\mathrm{C}$ infections among haemodialysis and peritoneal dialysis patients in Asia-Pacific countries: analysis of registry data. Nephrol Dial Transplant. 2009;24(5):1598-603. doi: 10.1093/ndt/gfn684. [PubMed: 19096083].

6. Igaki N, Nakaji M, Moriguchi R, Akiyama H, Tamada F, Oimomi M, et al. An outbreak of fulminant hepatitis $\mathrm{B}$ in immunocompromised hemodialysis patients. J Gastroenterol. 2003;38(10):968-76. doi: 10.1007/s00535-003-1180-1. [PubMed: 14614604].

7. Recommendations for Preventing Transmission of Infections
Among Chronic Hemodialysis Patients. MMWR Morb Mortal Wkly Rep. 2001;50:1-43.

8. Donati D, Gastaldi L. Controlled trial of thymopentin in hemodialysis patients who fail to respond to hepatitis B vaccination. Nephron. 1988;50(2):133-6. [PubMed: 3065661].

9. Kapoor D, Aggarwal SR, Singh NP, Thakur V, Sarin SK. Granulocytemacrophage colony-stimulating factor enhances the efficacy of hepatitis $B$ virus vaccine in previously unvaccinated haemodialysis patients. J Viral Hepat. 1999;6(5):405-9. [PubMed: 10607257].

10. Marangi AL, Giordano R, Montanaro A, De Padova F, Schiavone MG, Dongiovanni G, et al. Hepatitis B virus infection in chronic uremia: long-term follow-up of a two-step integrated protocol of vaccination. Am J Kidney Dis. 1994;23(4):537-42. [PubMed: 8154489].

11. Fabrizi F, Di Filippo S, Marcelli D, Guarnori I, Raffaele L, Crepaldi M, et al. Recombinant hepatitis B vaccine use in chronic hemodialysis patients. Long-term evaluation and cost-effectiveness analysis. Nephron. 1996;72(4):536-43. [PubMed: 8730417].

12. DaRoza G, Loewen A, Djurdjev O, Love J, Kempston C, Burnett S, et al. Stage of chronic kidney disease predicts seroconversion after hepatitis B immunization: earlier is better. Am J Kidney Dis. 2003;42(6):118492. [PubMed: 14655190].

13. Moher D, Liberati A, Tetzlaff J, Altman DG, Prisma Group . Preferred reporting items for systematic reviews and meta-analyses: the PRISMA statement. J Clin Epidemiol. 2009;62(10):1006-12. doi: 10.1016/j.jclinepi.2009.06.005. [PubMed: 19631508].

14. Poynard T, Conn HO. The retrieval of randomized clinical trials in liver disease from the medical literature. Controlled Clinical Trials. 1985;6(4):271-9. doi: 10.1016/0197-2456(85)90103-5. 
15. Viswanathan M, Ansari MT, Berkman ND, Chang S, Hartling L, McPheeters M, et al. Methods Guide for Effectiveness and Comparative Effectiveness Reviews. ; 2008. Assessing the Risk of Bias of Individual Studies in Systematic Reviews of Health Care Interventions.

16. DerSimonian R, Laird N. Meta-analysis in clinical trials. Controlled Clinical Trials. 1986;7(3):177-88. doi:10.1016/0197-2456(86)90046-2.

17. Higgins JP, Thompson SG, Deeks JJ, Altman DG. Measuring inconsistency in meta-analyses. BMJ. 2003;327(7414):557-60. doi: 10.1136/bmj.327.7414.557. [PubMed: 12958120].

18. Petitti DB. Approaches to heterogeneity in meta-analysis. Stat Med. 2001;20(23):3625-33. [PubMed: 11746342].

19. Galbraith RF. A note on graphical presentation of estimated odds ratios from several clinical trials. Stat Med. 1988;7(8):889-94. [PubMed: 3413368].

20. Klein S, Simes J, Blackburn GL. Total parenteral nutrition and cancer clinical trials. Cancer. 1986;58(6):1378-86. doi: 10.1002/10970142(19860915)58:6<1378::aid-cncr2820580635>3.0.co;2-s.

21. Benhamou E, Courouce AM, Jungers P, Laplanche A, Degos F, Brangier J, et al. Hepatitis B vaccine: randomized trial of immunogenicity in hemodialysis patients. Clin Nephrol. 1984;21(3):143-7. [PubMed: 6705277].

22. Aronoff GR, Maxwell DR, Batteiger BE, Fineberg NS. Hepatitis B virus vaccine: a randomized trial of a reduced dose regimen in hemodialysis patients. Am J Kidney Dis. 1985;6(3):170-2. [PubMed: 3898825].

23. Lelie PN, Reesink HW, de Jong-van Manen ST, Dees PJ, ReerinkBrongers EE. Immune response to a heat-inactivated hepatitis B vaccine in patients undergoing hemodialysis. Enhancement of the response by increasing the dose of hepatitis B surface antigen from 3 to 27 micrograms. Arch Intern Med. 1985;145(2):305-9. [PubMed: 3977491].

24. Seaworth B, Drucker J, Starling J, Drucker R, Stevens C, Hamilton J. Hepatitis $B$ vaccines in patients with chronic renal failure before dialysis. J Infect Dis. 1988;157(2):332-7. [PubMed: 2961818].

25. Bruguera M, Cremades M, Rodicio JL, Alcazar JM, Oliver A, Del Rio $\mathrm{G}$, et al. Immunogenicity of a yeast-derived hepatitis $B$ vaccine in hemodialysis patients. Am J Med. 1989;87(3A):30S-2S. [PubMed: 2528295].

26. Mitwalli A. Responsiveness to hepatitis B vaccine in immunocompromised patients by doubling the dose scheduling. Nephron. 1996;73(3):417-20. [PubMed: 8832600].

27. Mauri JM, Valles M. Effects of recombinant interleukin-2 and revaccination for hepatitis B in previously vaccinated, non-responder, chronic uraemic patients. Collaborative Group of Girona. Nephrol Dial Transplant. 1997;12(4):729-32. [PubMed: 9141002].

28. Deniz Ayli M, Ensari C, Ayli M, Mandiroglu F, Mut S. Effect of oral levamisole supplementation to hepatitis $\mathrm{B}$ vaccination on the rate of immune response in chronic hemodialysis patients. Nephron. 2000;84(3):291-2. [PubMed: 10720909].

29. McNulty CA, Bowen JK, Williams AJ. Hepatitis B vaccination in predialysis chronic renal failure patients a comparison of two vaccination schedules. Vaccine. 2005;23(32):4142-7. doi: 10.1016/j.vaccine.2005.03.020. [PubMed: 15913854].

30. Chow KM, Law MC, Leung CB, Szeto CC, Li PK. Antibody response to hepatitis B vaccine in end-stage renal disease patients. Nephron Clin Pract. 2006;103(3):c89-93. doi: 10.1159/000092016. [PubMed: 16534237].

31. Chow KM, Lo SH, Szeto CC, Yuen SK, Wong KS, Kwan BC, et al Extra-high-dose hepatitis B vaccination does not confer longer serological protection in peritoneal dialysis patients: a randomized controlled trial. Nephrol Dial Transplant. 2010;25(7):2303-9. doi: 10.1093/ndt/gfq094. [PubMed: 20185409].

32. Jadoul M, Goubau P. Is anti-hepatitis B virus (HBV) immunization successful in elderly hemodialysis (HD) patients? Clin Nephrol. 2002;58(4):301-4. [PubMed: 12400846].

33. Khedmat H, Aghaei A, Ghamar-Chehreh ME, Agah S. Sex bias in response to hepatitis $\mathrm{B}$ vaccination in end-stage renal disease patients: Meta-analysis. World J Nephrol. 2016;5(1):115-24. doi: 10.5527/wjn.v5.11.115. [PubMed: 26788471].

34. Fernandez E, Betriu MA, Gomez R, Montoliu J. Response to the hepatitis $B$ virus vaccine in haemodialysis patients: influence of malnutrition and its importance as a risk factor for morbidity and mortality. Nephrol Dial Transplant. 1996;11(8):1559-63. [PubMed: 8856211].

35. Navarro JF, Teruel JL, Mateos ML, Marcen R, Ortuno J. Antibody level after hepatitis $B$ vaccination in hemodialysis patients: influence of hepatitis C virus infection. Am J Nephrol. 1996;16(2):95-7. [PubMed: 8919223].

36. Ahuja TS, Kumar S, Mansoury H, Rodriguez H, Kuo YF. Hepatitis B vaccination in human immunodeficiency virus-infected adults receiving hemodialysis. Kidney Int. 2005;67(3):1136-41. doi: 10.1111/j.15231755.2005.00180.x. [PubMed: 15698455].

37. Alavian SM, Tabatabaei SV. The effect of diabetes mellitus on immunological response to hepatitis B virus vaccine in individuals with chronic kidney disease: A meta-analysis of current literature. Vaccine. 2010;28(22):3773-7. doi: 10.1016/j.vaccine.2010.03.038. [PubMed: 20371390].

38. Sennesael JJ, Van der Niepen P, Verbeelen DL. Treatment with recombinant human erythropoietin increases antibody titers after hepatitis B vaccination in dialysis patients. Kidney Int. 1991;40(1):121-8. [PubMed: 1921147].

39. Pol S, Legendre C, Mattlinger B, Berthelot P, Kreis H. Genetic basis of nonresponse to hepatitis B vaccine in hemodialyzed patients.J Hepatol. 1990;11(3):385-7. [PubMed: 2290031].

40. Jibani MM, Heptonstall J, Walker AM, Bloodworth LO, Howard AJ Hepatitis B immunization in UK renal units: failure to put policy into practice. Nephrol Dial Transplant. 1994;9(12):1765-8. [PubMed 7708261].

41. Mulley WR, Le ST, Ives KE. Primary seroresponses to double-dose compared with standard-dose hepatitis $\mathrm{B}$ vaccination in patients with chronic kidney disease: a systematic review and meta-analysis. Nephrol Dial Transplant. 2016 doi: 10.1093/ndt/gfv443. [PubMed: 26763670].

42. Chen YC, Su YC, Li CY, Wu CP, Lee MS. A nationwide cohort study suggests chronic hepatitis B virus infection increases the risk of end-stage renal disease among patients in Taiwan. Kidney Int 2015;87(5):1030-8. doi: 10.1038/ki.2014.363. [PubMed: 25426815].

43. Wu T, Li Y, Bian Z, Liu G, Moher D. Randomized trials published in some Chinese journals: how many are randomized? Trials 2009;10:46. doi: 10.1186/1745-6215-10-46. [PubMed: 19573242].

44. Moher D, Pham B, Jones A, Cook DJ, Jadad AR, Moher M, et al. Does quality of reports of randomised trials affect estimates of intervention efficacy reported in meta-analyses? Lancet. 1998;352(9128):60913. doi: 10.1016/S0140-6736(98)01085-X. [PubMed: 9746022].

45. Miller ER, Alter MJ, Tokars JI. Protective effect of hepatitis B vaccine in chronic hemodialysis patients. Am J Kidney Dis. 1999;33(2):356-60. [PubMed: 10023650].

46. Surquin M, Tielemans CL, Kulcsar I, Ryba M, Voros P, Mat O, et al Rapid, enhanced, and persistent protection of patients with renal insufficiency by ASO2(V)-adjuvanted hepatitis B vaccine. Kidney Int. 2010;77(3):247-55. doi: 10.1038/ki.2009.454. [PubMed:19940840].

47. Fabrizi F, Tarantino A, Castelnovo C, Martin P, Messa P. Recombinant Hepatitis B Vaccine Adjuvanted With ASO4 in Dialysis Patients: A Prospective Cohort Study. Kidney Blood Press Res. 2015;40(6):584-92. doi: 10.1159/000368534. [PubMed: 26566033]

48. Weinstein T, Chagnac A, Boaz M, Ori Y, Herman M, Zevin D, et al. Improved immunogenicity of a novel third-generation recombinant hepatitis $\mathrm{B}$ vaccine in patients with end-stage renal disease. Nephron Clin Pract. 2004;97(2):c67-72. doi: 10.1159/000078403. [PubMed: 15218332]. 